\title{
RESISTENCIA À TRAÇÃO (POR COMPRESSÃO DIAMETRAL) EM GESSOS ODONTOLÓGICOS: INFLUÊNCIA DA ESPESSURA DOS CORPOS DE PROVA
}

\author{
Professor Léo Werner Süffert \\ Professor Catedrático de Materiais \\ Dentários da Faculdade de Odonto- \\ logia, UFRGS; Chefe do Departa- \\ mento de Materiais Dentários. \\ Professor Ennio Pessoa \\ Professor Adjunto de Materiais Den- \\ tários da Faculdade de Odontologia, \\ UFRGS.
}

\section{SINOPSE}

Verificou-se a influência de diferentes espessuras em corpos de prova de gêsso (mantendo-se o diâ. metro igual) sôbre a sua resistência à tração, pelo método de compressão diametral11, observando-se que a variabilidade na repetição dos ensaios, ou seja, a reprodutibilidade dos ensaios é maior, nos corpos de prova de menor espessura, devendo-se, por êste motivo, dar preferência aos mesmos neste tipo de ensaio. Correlacionou-se ainda, os valôres de resistência à compressão, com os da resistência à tração, observando-se que a diferença entre as duas, diminue à medida que anmenta a espessura dos corpos de prova sub- metidos à compressão diametral, quando os resultados forem expressos em esfôrço. $\left(\mathrm{kg} / \mathrm{cm}^{2}\right)$

\section{INTRODUÇ̃̃}

Qualquer ensaio mecânico em qualquer material de uso odontológico, seja de emprêgo bucal ou no laboratório, possuirá realmente valor, somente se houver uma correlação en_tre o ensaio e a performance do material quando em uso.

Um dos ensaios mais comumente realizados com materiais odontológicos é o da resistência à compressão. Sabemos, no entanto, que tanto as pressões (ou compressões) exercidas durante a mastigação sôbre os mate- 
riais restauradores, quanto as pressões (ou compressões) exercidas sôbre os materiais de uso laboratorial (como é o caso da prensagem na ela_ boração de dentaduras, por exemplo), trazem como resultante a indução de esforços de cisalhamento e esforços de tração.

Os diversos métodos súgeridos para ensaios de tração (1), por sua vez, são passiveis de discussões e apresentam inconvenientes, particularmente em relação a.os materiais carentes de plasticidade.

Desde o surgimento do método Lobo Carneiro (2), minuciosamente analisado por Peltier (3) e, freqüente. mente referido na literatura internacional como «brazilian test», (6) em muitas investigações relacionadas com materiais Dentários $(4,5,6,7,8)$, se tem utilizado a «compressão diametral» na verificação da resistência à tração. Inclusive, a mais recente modificação da Especificação $\mathrm{N}^{\circ} 1 \mathrm{da}$ American Dental Association (9), para Ligas para Amálgamas Odontológicos, em seu requisito 3.4 estipula um valor mínimo para a resistência à tração das referidas ligas, por compres. são diametral.

Na quase totalidade das investigações relacionadas com ensaios de tracão por compressão diametral, os corpos de prova utilizados, apresentam forma e dimensões idênticas às normalmente empregadas em ensaios para a verificação de resistência à com_ pressão. Isto, no entanto, não ocorre na investigação de Earnshaw e Smith (10), os quais para ensaios de compressão diametral utilizaram discos circulares com diâmetro de 1,0 pole- gada $(25,4 \mathrm{~mm})$ e espessura de 0,5 polegadas $(12,7 \mathrm{~mm})$.

Êste fato levou-nos ao objetivo principal do presente trabalho, qual seja o de: $1^{\circ}$ ) Verificar a influência de diferentes espessuras em corpos de prova de gêsso (mantendo-se o diâmetro igual) sôbre sua resistência à tração, pelo método de compressão diametral (11).

$2^{\circ}$ ) Verificar a relação entre a re. sistência à tração assim obtida e a correspondente resistência à compressão.

\section{MATERIAIS E MÉTODOS}

Utilizamos dois tipos de gêssos: um gêsso tipo pedra (marca HERODENT, fabricação Herman Josias S. A. Ind. e Com., Rio de Janeiro) e um gêsso tipo «especial» (marca VEL-MIX, fabricação Kerr Dental Mfg. Company, Detroit, Michigan, USA).

Êstes gêssos foram selecionados por serem dos tipos pedra e especial, os mais empregados pelo cirurgiãodentista brasileiro.

A relação água/pó utilizada, foi aquela correspondente à «consistência para ensaios», segundo as normas propostas para «Produtos à ba_ se de gypsum» pela Associação Dentária Americana (12), sendo respectivamente para o Gêsso HERODENT $=0,40$ e para o Gêsso VELMIX $=0,25$.

A técnica de espatulação utilizada foi a mecânico-manual, correspondendo à 50 voltas da manivela, executadas em 20 segundos.

o preenchimento dos moldes era 
executado sob vibração padronizada por uma técnica que reduzisse ao mínimo a possibilidade de formação de bôlhas ou poros nos coppos de prova resultantes.

Os corpos de prova consistiam, con_ forme se pode observar pela Fig. $\mathrm{N}^{\circ} 1$, cilindros de três tipos, com diâmetros idênticos $(2,515 \mathrm{~cm})$ e espessuras (ou alturas) diferentes e aos quais passamos a denominar respectivamente de:

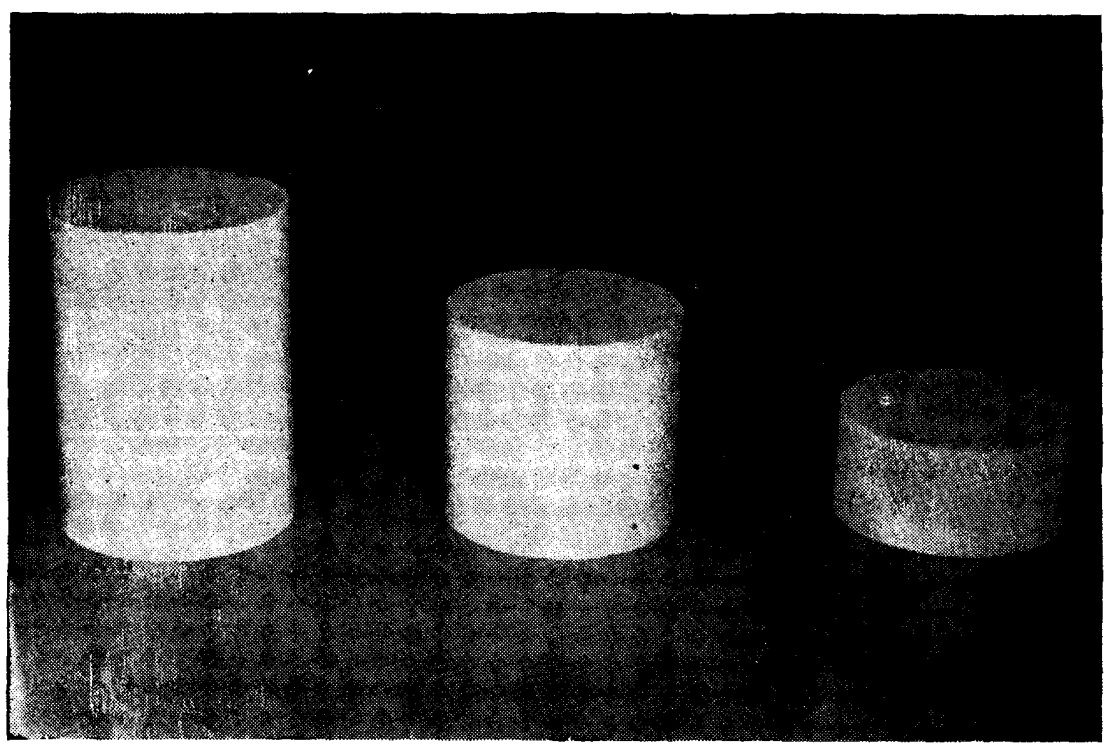

Fig. 1

Três comprimentos distintos de Corpos de Prova utilizados nos ensaios de tração, por compreensão diametral: C.P. Longos, C.P. Médios e C.P. Curtos

\section{DIÂMETRO}

$\begin{array}{ll}\text { CURTOS } & 2,515 \mathrm{~cm} \mp 0,005 \mathrm{~cm} \\ \text { MEDIOS } & 2,515 \mathrm{em} \pm 0,005 \mathrm{~cm} \\ \text { LONGOS } & 2,515 \mathrm{~cm} \mp 0,005 \mathrm{~cm}\end{array}$

Nos ensaios para o relacionamento com a resistência à compressão,
ESPESSURA

$$
\begin{aligned}
& 1,235 \mathrm{~cm} \mp 0,024 \mathrm{~cm} \\
& 2,425 \mathrm{~cm} \mp 0,059 \mathrm{~cm} \\
& 3,591 \mathrm{~cm} \mp 0,046 \mathrm{~cm}
\end{aligned}
$$

utilizamos corpos de prova com as seguintes dimensões: 
DIÂMETRO

$1,350 \mathrm{~cm} \mp 0,004 \mathrm{~cm}$
ESPESSURA

$2,521 \mathrm{~cm} \mp 0,011 \mathrm{~cm}$
As condições de temperatura ambiente e umidade relativa, durante a elaboração dos corpos de prova

UMIDADE RELATIVA: TEMPERATURA · AMBIENTE:

Como nosso objetivo era o de ve_ rificar a resistência sêca dos gêssos nos diversos ensaios e queríamos evitar a influência de variações (ainda que pequenas) de umidade relativa e temperatura ambientes, colocávamos os corpos de prova, tão logo atingiam a sua prêsa final, numa estufa à $37^{\circ} \mathrm{C}$, onde permaneciam pelo espaço de 24 horas, permitindo-se sua volta à temperatura e ruptura dos mesmos, foram as seguintes:

$59 \mp 9 \%$

$25 \mp^{\circ} \mathrm{C}$

ambiente imediatamente antes da realização dos ensaios.

A comprovação de que o espaço de tempo de 24 horas era suficiente para a eliminação do excesso de água das misturas, foi obtida através pesagens sucessivas, nos diversos corpos de prova que permaneciam por tempos diferentes na estufa à $37^{\circ} \mathrm{C}$, conforme se pode observar na Tabela $\mathrm{N}^{\circ} 1$

\section{TABELA Nי 1}

PESAGENS SUCESSIVAS DOS CORPOS DE PROVA DE GESSO APOS PERMANENCIA NA ESTUFA A $37^{\circ} \mathrm{C}$, POR DIFERENTES ESPACOOS DE TEMPO. (Resultados em gramas)

\begin{tabular}{l|c|c|c|c|c}
\hline & Início & 1 Hora & 5 Horas & 24 Horas & 96 Horas \\
\hline \multirow{3}{*}{ CURTOS } & 10,9615 & 10,4070 & 9,3850 & 9,3485 & 9,3470 \\
& 10,9745 & 10,3820 & 9,3690 & 9,3600 & 9,3580 \\
& 11,1270 & 10,5570 & 9,5360 & 9,5080 & 9,5070 \\
\hline \multirow{2}{*}{ MEDIOS } & 21,6105 & 20,8075 & 18,9440 & 18,3530 & 18,3500 \\
& 21,2190 & 20,3665 & 18,4515 & 18,0300 & 18,0275 \\
& 21,6110 & 20,7235 & 18,7655 & 18,3680 & 18,3660 \\
\hline \multirow{3}{*}{ LONGOS } & 32,1910 & 31,2240 & 28,6800 & 27,4155 & 27,4120 \\
& 32,0500 & 31,2540 & 28,4800 & 27,4900 & 27,4880 \\
\hline
\end{tabular}

R. Fac. Odont. P.A. 
Os ensaios de resistência à tração por (compressão diametral) bem como os ensaios de resistência à compressão foram realizađos numa máquina de Ensaio Universal Modêlo LLOS», Losenhausenwerk, Düsseldorfer Maschinenbau A. G., tipo UHP/ ZHP 2, sendo a velocidade de aplicação de carga de $225 \mathrm{~kg} / \mathrm{min}$ e os resultados que adiante apresentamos, constituem média de no mínimo 20 ensaios para cada variável. Os resultados foram submetidos à análise de variância.

o cálculo para os esforços, nos ensaios de tração, foram realizados, segundo a representação esquemática ca da Fig. 2, em que $\mathbf{T}=$ esfôrço em $\mathrm{kg} / \mathrm{cm} 2 ; \mathrm{D}=$ diâmetro $\mathrm{em} \mathrm{cm}$ e $\mathrm{t}=$ espessura ou altura $\mathrm{em} \mathrm{cm}$.
A

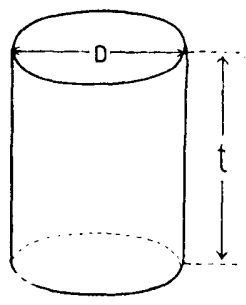

B

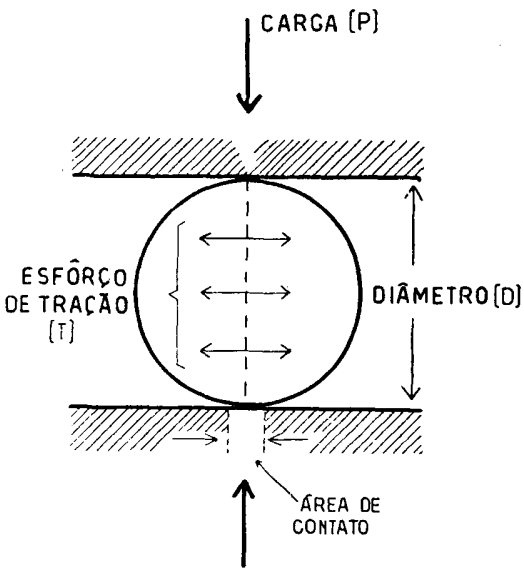

$T=\frac{2 P}{\pi D t}$

Fig. 2

Representação esquemática do ensaio de tração por compressão diametral. $\mathrm{T}=$ esforco em $\mathrm{Kg} / \mathrm{cm}^{2} ; \quad D=$ diametro $\mathrm{em} \mathrm{cm} ; \mathrm{t}=$ espessura ou altura em $\mathrm{cm}$.

A Fig. 3, mostra um corpo de prova do tipo MeDIO, sendo submetido ao ensaio de tração, por compressão diametral.

Na Fig. 4, podemos observar rup- turas típicas, dos três tipos de corpos de prova, LONGOS, MEDIOS e CURTOS, submetidos ao referido ensaio.

Eventualmente algum resultado, 
apresentava-se totalmente discrepante dos demais. As linhas de ruptura não acompanhavam os diâmetros dos corpos de prova, mas eram tortuosas ou curvas. Nestas oportunidades, constatávamos, invariàvelmente, a existência de bôlhas de ar, para onde se dirigiam as rupturas, conforme podemos, observar na
Fig. de $\mathrm{N}^{\circ}$ 5. Quando a discrepância ultrapassava $o$ valor máximo estipulado, segundo o item 4.3.5.1 da Especificação $N^{\circ} 11$ aceita pelo Grupo Brasileiro de Materiais Dentários (13) o corpo de prova era detidamente examinado e, constatadas as bôlhas existentes, era eliminado.

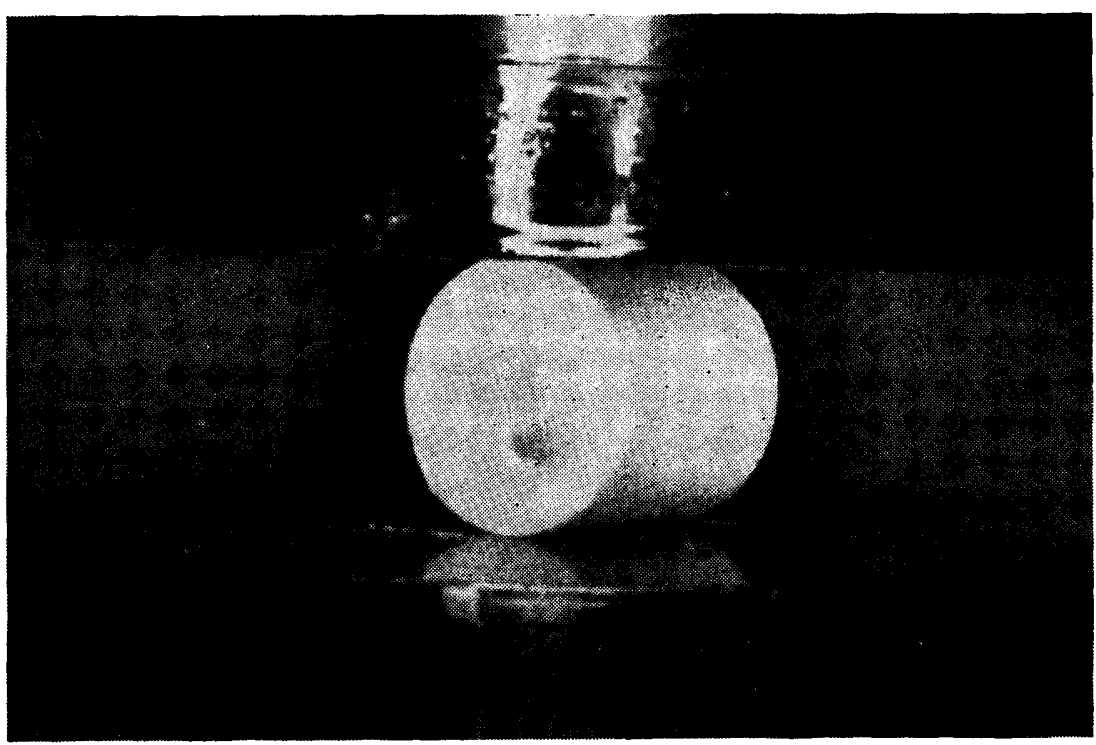

Fig. 3

Corpo de Prova de comprimento Médio, sendo submetido ao ensaio de tração por compressão diametral. 


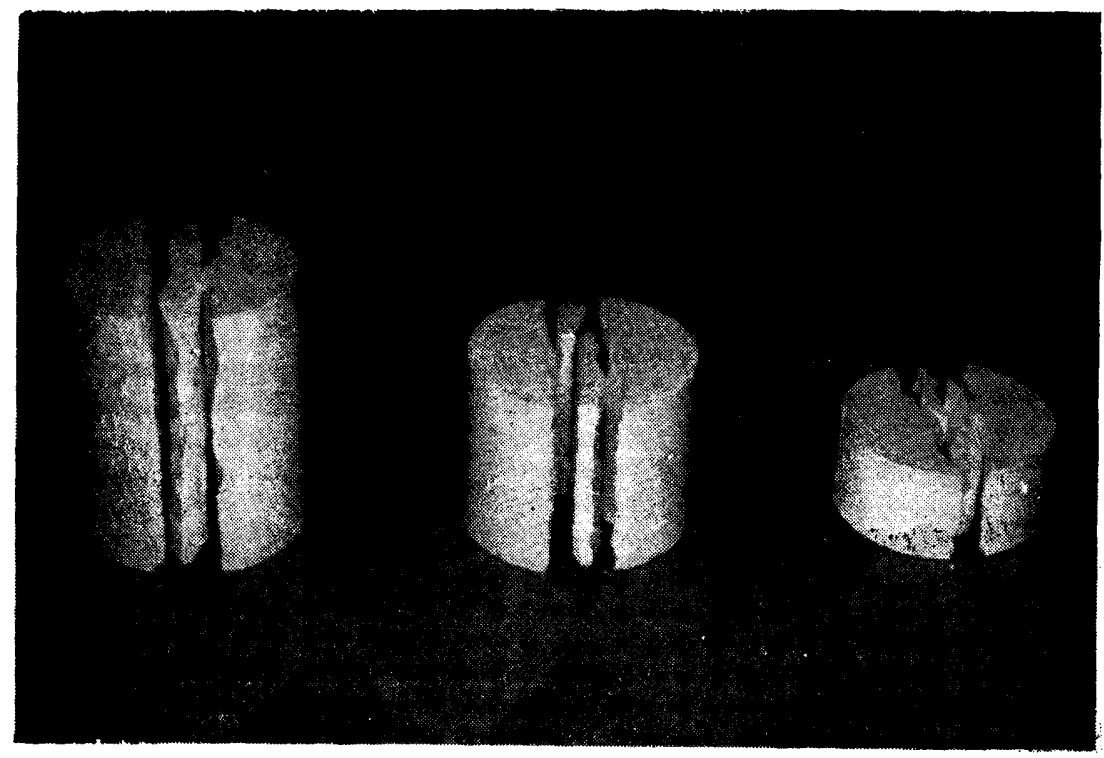

Fig. 4

Rupturas típicas dos C.P. submetidos aos ensaios de tração pelo Método de Lobo Carneiro. 


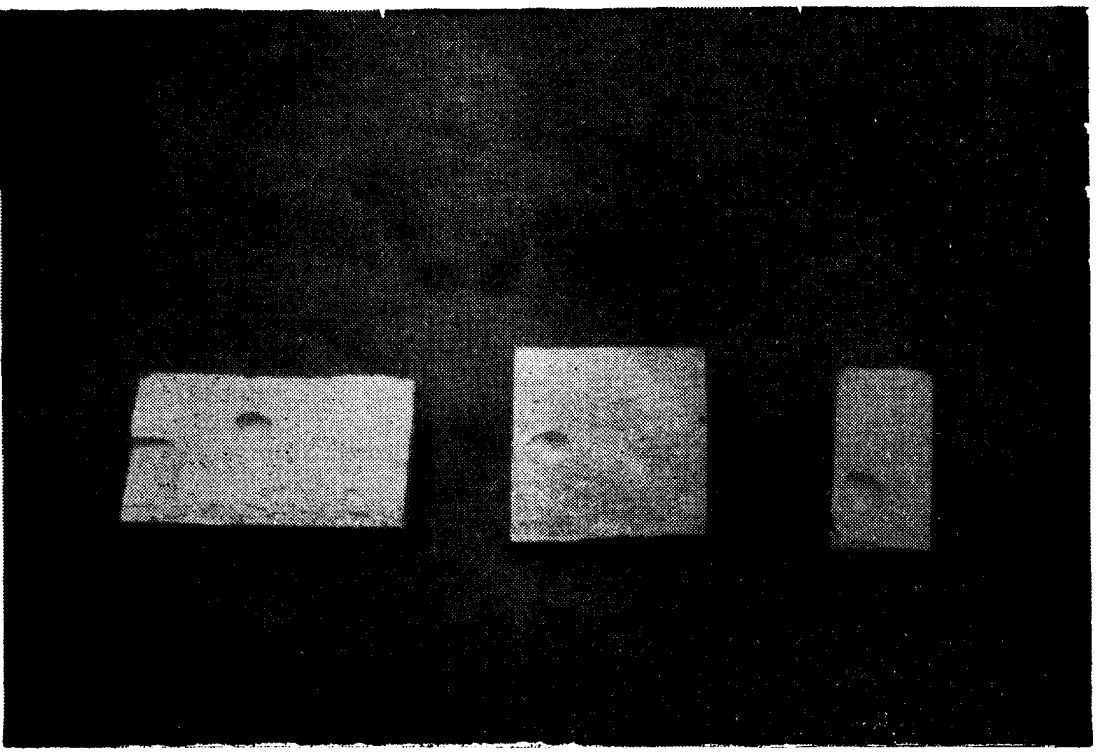

Fig. 5

Corpos de Prova defeituosos, submetidos aos ensaios de tração por compressão diametral. Valôres totalmente discrepantes de cargas de ruptura indicavam, quase que sistematicamente, a presença de enormes bôlhas no interior dos C.P., semelhantes as que se podem observar na figura. 


\section{RESULTADOS}

Os resultados obtidos e, expressos em valôres de esforços, podem ser observados no Gráfico de $\mathrm{N}^{\circ}$ I e Tabela $\mathrm{N}^{\circ} 2$.

Uma outra expressão dêstes mesmos resultados, ou seja através cargas de ruptura, é apresentada no
Gráfico de $\mathrm{N}^{\circ}$ II e Tabela $\mathrm{N}^{0} 3$.

A diferença de variabilidade dentro dos ensaios à que submetemos os corpos de prova CURTOS, MÉdIOS e LONGOS dos gêssos HERODENT e VEL-MIX, poderá ser observada no Gráfico III, cujos resultados foram submetidos à análise estatística, segundo Tabela $\mathrm{N}^{0} 4$.

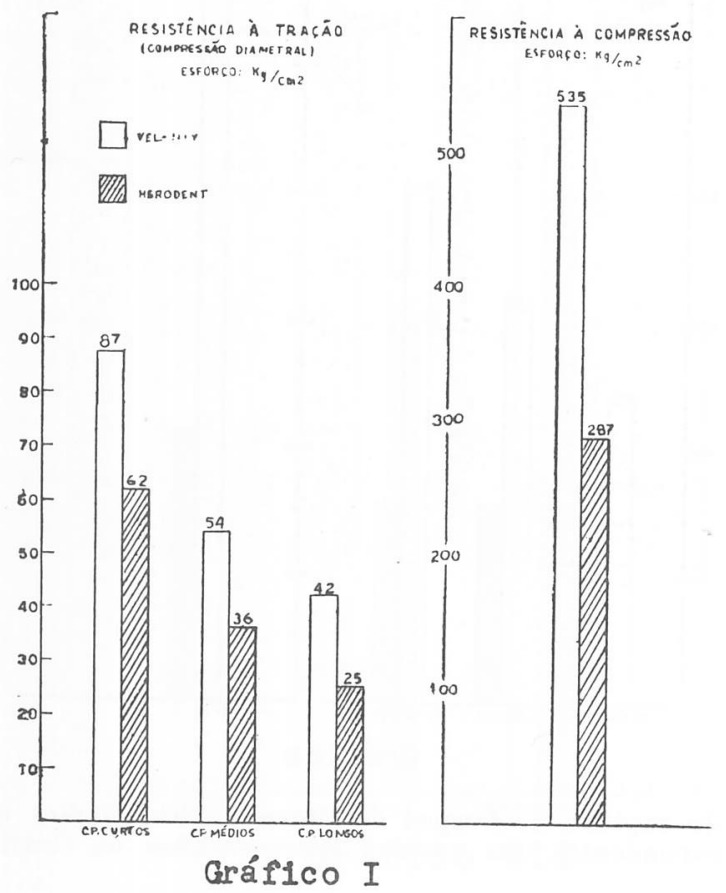

Resistência à tração de duas marcas de Gêsso Pedra, pelo método Lobo Carneiro (Compressão Diametral) em função de diversos comprimentos de Corpos de Prova. Ao lado, para fins de comparação, as respectivas resistệncias à compressão. 


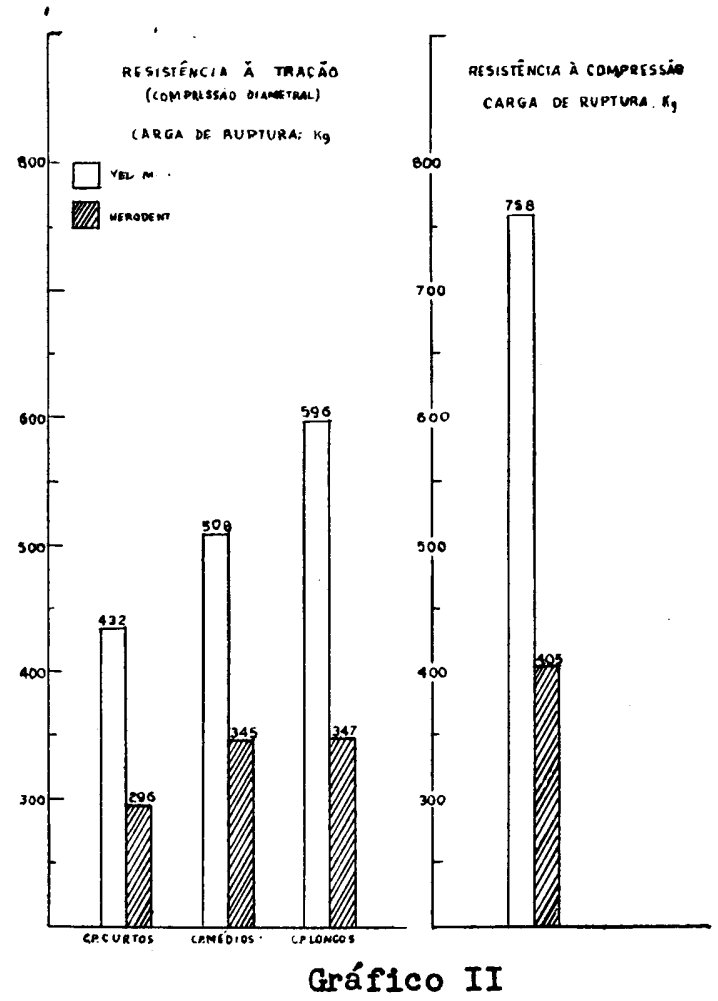

Cargas de ruptura de Corpos de Prova Curtos, Médios e Longos, correspondentes aos ensaios representados no Gráfico I. 


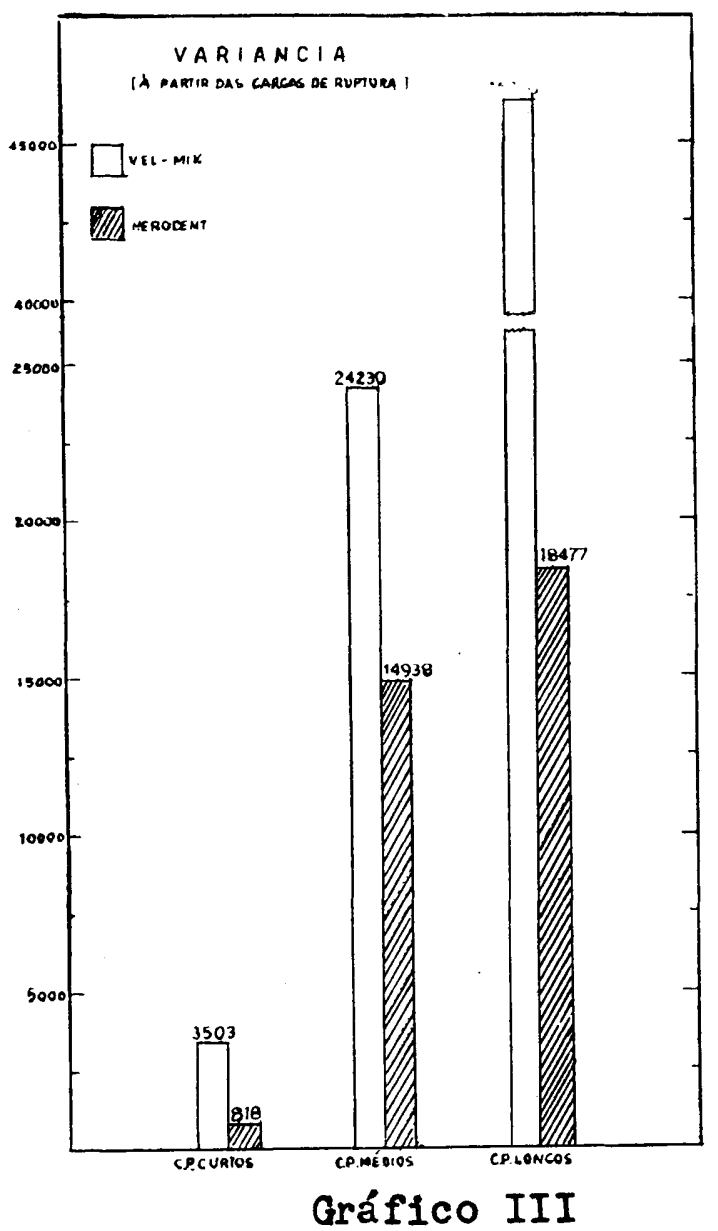

Representação gráfica da Análise da Variância dos experimentos correspondentes ao Gráfico II, em que se observa a MENOR variabilidade nos ensaios dos C. P. Curtos, quando comparados com os Médios e Longos. A diferença é estatìsticamente significativa ao nível de $1 \%$. 


\section{TABELA N $N^{\circ}$}

\section{$\begin{array}{llllllll}\text { E S F } & \boldsymbol{O} & \mathbf{R} & \mathbf{C} & \mathbf{O} & \left(\mathrm{Kg} / \mathrm{cm}^{2}\right)\end{array}$}

VEL - M I X

\begin{tabular}{l|c|c|c|c}
\hline & Curtos & Médios & Longos & Compressão \\
\hline$\overline{\mathbf{x}}$ & 87 & 54 & 42 & 535 \\
$\mathbf{s}^{2}$ & 131,94 & 274,58 & 238,05 & $5.523,42$ \\
$\mathbf{s}$ & 11,48 & 16,57 & 15,43 & 74,32 \\
$\mathrm{CV}$ & $7,50 \%$ & $30,68 \%$ & $36,74 \%$ & $13,89 \%$ \\
$\mathrm{e}$ & 1,039 & 3,71 & 3,45 & 16,62 \\
\hline
\end{tabular}

H E R O D E N T

\begin{tabular}{l|c|c|c|c}
\hline & Curtos & Médios & Longos & Compressão \\
\hline$\overline{\mathrm{x}}$ & 62 & 36 & 25 & 287 \\
$\mathbf{s}^{2}$ & 21,63 & 163,05 & 95,31 & $1.742,31$ \\
$\mathrm{~s}$ & 4,651 & 12,769 & 9,763 & 41,74 \\
$\mathrm{CV}$ & $7,50 \%$ & $35,47 \%$ & $39,05 \%$ & $14,54 \%$ \\
$\mathrm{e}$ & 1,039 & 2,855 & 2,182 & 9,333 \\
\hline
\end{tabular}

R. Fac. Odont. P.A. 
TABELA $N^{\bullet} 3$

$\begin{array}{llllllllllll}\text { C A R G A S } & \text { D E } & \text { R U P T U R A } & \text { (Kg) }\end{array}$

VE L - M I X

\begin{tabular}{l|c|c|c|c}
\hline & Curtos & Médios & Longos & Compressão \\
\hline$\tilde{\mathbf{x}}$ & 432 & 508 & 596 & 758 \\
$\mathrm{~s}^{2}$ & $3.503,22$ & $24.229,68$ & $46.306,37$ & $12.054,00$ \\
$\mathrm{~s}$ & 59,18 & 155,66 & 215,19 & 109,79 \\
$\mathrm{CV}$ & $13,70 \%$ & $30,64 \%$ & $36,10 \%$ & $14,48 \%$ \\
$\mathrm{e}$ & 13,58 & 34,80 & 48,12 & 24,55 \\
\hline
\end{tabular}

H E R O D E N T

\begin{tabular}{l|c|c|c|c}
\hline & Curtos & Médios & Longos & Compressão \\
\hline$\overline{\mathrm{x}}$ & 296 & 345 & $\mathbf{3 4 7}$ & 405 \\
$\mathrm{~s}^{2}$ & 818 & 14.938 & 18.477 & 4.006 \\
$\mathrm{~s}$ & 28,60 & 122,20 & 135,93 & 63,30 \\
$\mathrm{CV}$ & $9,66 \%$ & $35,42 \%$ & $39,17 \%$ & $15,63 \%$ \\
$\mathrm{e}$ & 6,395 & 27,33 & 30,39 & 14,15 \\
\hline
\end{tabular}

R. Fac. Odont. P.A. 
HERODENT - RESISTENCCIA A TRAÇÃO (ESFÔRÇO: $\mathrm{kg} / \mathrm{cm}^{2}$ ) ANÁLISE DA VARIANCIA

(Gráfico I e Tabela 2)

\begin{tabular}{l|c|c|c|c}
\hline FONTE & $\cdot \mathbf{S Q}$ & $\mathbf{G L}$ & $\mathbf{Q M}$ & $\mathbf{F}_{\mathrm{c}}$ \\
\hline Tratamento & 14.343 & $\mathrm{k}-1=2$ & 7.171 & $\mathbf{7 6 , 8 3 7}$ \\
\hline Er r o & 5.320 & $\mathrm{n}-\mathrm{k}=57$ & 93 & \\
\hline TOTAL & 19.663 & $\mathrm{n}-1=59$ & & \\
\hline
\end{tabular}

VEL-MIX - RESISTENCIA A TRAÇÃO (ESFÔRÇO: $\mathrm{kg} / \mathrm{cm}^{2}$ )

ANÁLISE DA VARIÂNCIA

(Gráfico I e Tabela 2)

\begin{tabular}{l|c|c|c|c}
\hline FONTE & $\mathbf{S Q}$ & $\mathbf{G L}$ & $\mathbf{Q M}$ & $\mathbf{F}_{\mathbf{c}}$ \\
\hline Tratamento & 21.235 & $\mathrm{k}-1=2$ & 10.617 & 49,152 \\
\hline E r r o & 12.115 & $\mathrm{n}-\mathrm{k}=56$ & 216 & \\
\hline TOTAL & 33.350 & $\mathrm{n}-\mathrm{l}=58$ & & \\
\hline
\end{tabular}

HERODENT - RESISTENCIA A TRAÇÃO (CARGA DE RUPTURA: kg) ANÁLITSE DA VARIÂNCIA

(Gráfico II e Tabela 3)

\begin{tabular}{l|c|c|c|c}
\hline FONTE & $\mathbf{S Q}$ & $\mathbf{G L}$ & $\mathbf{Q M}$ & $\mathbf{F}_{\mathbf{c}}$ \\
\hline Tratamento & $\mathbf{3 3 . 6 1 3}$ & $\mathrm{k}-\mathrm{l}=\mathbf{2}$ & $\mathbf{1 6 . 8 0 6}$ & $\mathbf{1 , 4 7 2}$ \\
\hline Err o & 650.424 & $\mathrm{n}-\mathrm{k}=57$ & 11.411 & \\
\hline TOTAL & 684.037 & $\mathrm{n}-\mathrm{l}=\mathbf{5 9}$ & & \\
\hline
\end{tabular}

VEL-MIX - RESISTENCIA A TRAÇÃO (CARGA DE RUPTURA: kg) ANÁLISE DA VARIÂNCIA

(Gráfico II e Tabela 3)

\begin{tabular}{l|c|c|c|c}
\hline FONTE & SQ & GL & QM & F $_{\mathrm{c}}$ \\
\hline Tratamento & 261.675 & $\mathrm{k}-1=5$ & 130.837 & 5,221 \\
\hline Er r o & 1.403 .243 & $\mathrm{n}-\mathrm{k}=56$ & 25.058 & \\
\hline TOTAL & 1.664 .918 & $\mathrm{n}-\mathrm{l}=58$ & & \\
\hline
\end{tabular}




\section{INTERPRETAÇAO DOS RESULTADOS}

\subsection{MEDIAS}

1. Considerando-se a resistência a tração expressa pela fórmula com- pensada de Lobo Carneiro $(2,3,11)$ e, submetidos à análise estatística os resultados obtidos com os corpos de prova CURTOS, MEDIOS e LONGOS dos gêssos HERODENT e VEL-MIX, conforme Gráfico I e Tabelas 2 e 4, observamos as seguintes diferenças:

\subsection{HERODENT}

1.1.1 CURTOS E MÉDIOS: diferença muito significativa (.01)

1.1.2 CURTOS e LONGOS: diferença muito significativa (.01)

1.1.3 MEDIOS e LONGOS: diferença muito significativa (.01)

\subsection{VEL-MIX}

1.2.1 CURTOS e MÉDIOS: diferença muito significativa (.01)

1.2.2 CURTOS e LONGOS: diferença muito significativa (.01)

1.2.3 MEDIOS e LONGOS: diferença significativa (.05)

2. Considerando-se a resistência à tração (por compressão diametral), expressa simplesmente por cargas de ruptura, conforme Gráfico II e Tabela 3 e 4, observamos que:

2.1 HERODENT: não há diferenças significativas entre as médias dos corpos de prova CURTOS, MEDIOS e LONGOS.

2.2 VEL-MIX: há diferença significativa ao nível de $1 \%$, apenas entre os corpos de prova CURTOS e LONGOS.

\subsection{RELACAO ENTRE A RESIS. TENCLA A TRACXO E RE- SISTENCIA A COMPRESSAO}

1. Comparando-se a resistência à compressão com a resistência à tração dos gêssos Herodent e Vel-Mix, computando os resultados de cargas de ruptura e esforços, conforme Gráficos I e II e Tabelas 2 e 3, encontramos as seguintes percentagens ou frações: 
CARGAS DE RUPTURA

VEL-MIX

CURTOS: $56,99 \%(1 / 1,75)$

MEDIOS: $67,02 \%(1 / 1,49)$

LONGOS: $78,63 \%(1 / 1,27)$

\section{ESFORCYO}

VEL-MIX

CURTOS: $16,26 \%(1 / 6,15)$

MÉDIOS: $10,09 \%(1 / 9,91)$

LONGOS: $7,85 \%(1 / 12,74)$

\subsection{VARIÂNCLAS}

1. A diferença de variabilidade na repetição dos ensaios entre os corpos de prova CURTOS, MEDIOS e LONGOS, com os dois gêssos pes-
HERODENT

CURTOS: $73,09 \%(1 / 1,37)$

MEDIOS: $85,18 \%(1 / 1,17)$

LONGOS: $85,68 \%(1 / 1,17)$

\section{HERODENT}

\author{
CURTOS: $(21,60 \% \quad(1 / 4,63)$ \\ MÉDIOS: $12,54 \%(1 / 7,97)$ \\ LONGOS: $8,71 \%(1 / 11,48)$
}

quisados (HERODENT e VEL-MIX) foi por nós estudada através da ANÁLISE DA VARIÂNCIA, a partir das cargas de ruptura, conforme os dados do Gráfico III e Tabelas 3 e 4.

\subsection{HERODENT}

1.1.1 CURTOS e MéDIOS: difereņa muito significativa (.01)

1.1.2 CURTOS e LONGOS: diferença muito significativa (.01)

1.1.3 MÉDIOS e LONGOS: diferença não significativa

\subsection{VEL-MIX}

1.2.1 CURTOS e MEDIOS: diferença muito significativa (.01)

1.2.2 CURTOS e LONGOS: diferença muito significativa (.01)

1.2.3 MEDIOS e LONGOS: diferença não significativa

\section{CONCLUSÕES}

5.1 As diferenças das médias, entre os corpos de prova de diferentes espessuras, quando submetidos ao ensaio de tração por compressão diametral, são muito significativas, quando os resultados forem expressos em esfôrço. As diferenças não são significativas (exceto num caso), quando os resultados forem expressos simplesmente em cargas de ruptura.

5.2 Relacionando os valôres de resistência à compressão com os de resistência à tração, observamos que a diferença entre as duas, nos Gêssos por nós pesquisados, diminue à

R. Fac. Odont. P.A. 
medida que aumenta a espessura dos corpos de prova submetidos à compressão diametral. (resultados expressos em esfôrço: $\mathrm{kg} / \mathrm{cm} 2$ ).

5.3 A diferença de variabilidade na repetição dos ensaios, ou seja, a reprodutibilidade dos ensaios com os corpos de prova de diferentes espessuras, para a verificação da resistência à tração (por compressão diametral) indica que se deve dar preferência aos corpos de prova de menor espessura, ou seja, os mais curtos.

OBSERVAC̣̃̃ 0: A explicação para a conclusão 5.3, em nosso entender, reside na ausência de homogeneidade dos materiais utilizados em Odontologia, os quais submetidos à compressão diametral apresentam em estudos de foto-elasticidade orientação das bandas de tensão totalmente diferente. No caso de gêssos, nos corpos de prova de menor espessura, as possibilidades de bôlhas e poros, durante a vibração e preenchimento dos moldes, seria bem menor.

\section{SUMLARY}

The influence of different specimen thicknesses on the tensile strenght (by diametral compression method 11) of dental stones has been studdied. It was observed that there was greater reproducibility of results as the thickness of the samples decreased. This leads us to recommend the latter type of samples, when conducting diametral compression tensile strenght tests.

A relationship between the compression strenght and tensile strenght was also studdied. It was found that the difference between the two strenghts decreases as the thickness of the diametral compression strenght samples increases. The results were expressed in stress values.

\section{REFERÊNCIAS BIBLIOGRÁFICAS}

1 - PETRUCCI, Eladio. Ensaio de qualificação de cimento: determinação da resistência mecânica. São Paulo, Ass. Bras. de Cimento Portland, 1959. $85 \mathrm{p}$.

2 - CARNEIRO, F. L. \& BARCELLOS, A. Tensile strenghts of concret. R.I.L.E.M. Bull., 13: 97-107, Mar. 1953.

3 - PELTIER, R. Theoretical investigation of the Brazilian Test. R.I. L.E.M. Bulle, 19: 26-69, Nov. 1954 .

4 - SWEENEY, W. T. \& BURNS, C. L. Application of the diametral compression test to dental materials. I.A.D.R. Abstracts, 43: 122, Jul. 1965 .

5 - RUDNICK, A. et alii - An analysis of the diametral compression test. Matls. Res. \& Standards. Apr. 1963. p. 283.

6 - KORAN, A. \& ASGAR, K. A comparison of dental amalgams made from a spherical alloy and from a comunited alloy. J. Amer. Dent. Asses Chicago, 75(4): 912-917, Oct. 1967. 
7 - JOÃO, Mario \& CHEVITARESE, Orlando. Relação entre a resistência à compressão e à resistência à tração de gêsso. Odont. Atual, Rio de Janeiro, 1(1): 23-26, Dez. 1968.

8 - NAGAI, Kazuo et alii. Studies on the tensile strenght of dental amilgams by the application of diametral compression. J. Nihon Univ. sch. Dent., Tokyo, 12(1): 9-24, Mar. 1970.

9 - COUICIL ON DENTAL MATERIALS AND DEVICES. American Dental Association Specification n. 1, revised (Effective: june, 1970), J. Amer.'Dent. Ass,, Chicago, 79: 1206-1209, nov. 1969.

10 - EARSHAN, R. \& SMITH, D. C. The tensile and compressive strenght of plaster and stone. Aust, D. Jo, Sidney, 11(6): 415-422, dec. 1966.

11 - ASTM STANDARDS. Tentative method of test for splitting tensile strenght of molded concret cylinders. ASTM designation C-49664T, ASTM STANDARDS, 1964, part 10, p. 351.

12 - COUNCIL ON DENTAL MATERIALS AND DEVICES (ADA) Proposed A.D.A. Specification for dental Gypsum products. June, 1969, DMG, IADR.

13 - GRUPO BRASILEIRO DE MATERIAIS DENTARIOS, 11. Especificação para revestimentos para fundições de liga de ouro odontológicas, GBMD, Pôrto Alegre, RS, 1966. p. 1-5.

14 - LAUTENSChlager, E. P. \& HARCOURT, J. K. Photoelastic observation in diametral compression testing. J. Dent. Res., Chicago, 49(1): 175, Jan.-Feb., 1970. 